\title{
Plasmonic Nanolayer Composites: Coupled Plasmon Polaritons, Effective-Medium Response, and Subdiffraction Light Manipulation
}

\author{
Justin Elser, ${ }^{1}$ Alexander A. Govyadinov, ${ }^{1}$ Ivan Avrutsky, ${ }^{2}$ Ildar Salakhutdinov, ${ }^{2}$ and Viktor A. Podolskiy ${ }^{1}$ \\ ${ }^{1}$ Department of Physics, Oregon State University, 301 Weniger Hall, Corvallis, OR 97331-6507, USA \\ ${ }^{2}$ Department of Electrical and Computer Engineering, Wayne State University, Detroit, MI 48202, USA
}

Received 14 February 2007; Accepted 4 June 2007

Recommended by Christian Brosseau

We analyze the evolution of the modes in nanoplasmonic multilayered structures and study the transition of the optical properties of these systems to the effective-medium regime. We derive the effective-medium parameters and study the validity of our analytical results with exact numerical solutions of Maxwell equations. Finally, we explore the applications of multilayered systems for subwavelength light confinement in planar and circular waveguides.

Copyright (C) 2007 Justin Elser et al. This is an open access article distributed under the Creative Commons Attribution License, which permits unrestricted use, distribution, and reproduction in any medium, provided the original work is properly cited.

\section{INTRODUCTION}

A number of exciting applications in single-molecule imaging, spectroscopy, high-resolution lithography, and alloptical computing may benefit from light confinement and manipulation in $\mathrm{nm}$-scale areas. Unfortunately, the diffraction limit prevents confinement of free-space optical radiation to dimensions smaller than $\sim \lambda_{\text {in }} / 2$, with $\lambda_{\text {in }}=\lambda_{0} /|n|=$ $c /(\omega|n|)$ being the wavelength inside the material, $c, \lambda_{0}, \omega$ being speed of light, wavelength, and angular frequency of radiation in vacuum, respectively, and $n$ being the index of refraction. Conventional resolution-improvement techniques involve either increase of operating frequency (deep UV lithography) $[1,2]$ or near-field operations (scanning near-field optical microscopy) $[3,4]$. Another approach to increase the resolution is based on operations with highindex media. While a significant improvement of resolution can be achieved with natural materials (Si photonics) $[5,6]$, a further progress of subdiffraction light manipulation requires the design of metamaterials [7-21] with even higher refractive indices. In this work, we present an analytical and numerical analysis of perspectives of ultra-high index metamaterials based on plasmonic nanolayer structures for light manipulation in the areas smaller than $\lambda_{0} / 10$ at $\mathrm{UV}$, visible, IR, and $\mathrm{THz}$ frequencies.

The dielectric properties of plasmonic media are strongly affected by the dynamics of their free charges. In particular, the permittivities of noble metals and highly doped semiconductors are negative for frequencies below their plasma frequencies (plasma frequencies of noble metals are of the order of $10 \mathrm{eV}$, yielding negative permittivities across parts of the UV, visible, and IR ranges $[22,23])$. An interface between materials with negative and positive dielectric permittivities may support a special kind of electromagnetic wave, known as a surface plasmon polariton (SPP) [24]. The effective index of this wave can be related to the component of its propagating constant along the propagation direction $k_{z}$ (Figure 1) and permittivities of plasmonic (metallic) and dielectric materials $\epsilon_{m}$ and $\epsilon_{d}<\left|\epsilon_{m}\right|$ via

$$
n_{\mathrm{SPP}}=\operatorname{Re}\left(\frac{k_{z} c}{\omega}\right)=\operatorname{Re}\left(\sqrt{\frac{\epsilon_{m} \epsilon_{d}}{\epsilon_{m}+\epsilon_{d}}}\right) .
$$

Note that since $n_{\mathrm{SPP}}^{2}>\epsilon_{d}, \epsilon_{m}$, the field of an SPP exponentially decays into both the metal and dielectric media. However, since in reality for optical frequencies $\left|\epsilon_{m}\right| \gg \epsilon_{d}$, the index of SPP wave only slightly exceeds that of the dielectric $n_{d}=\sqrt{\epsilon_{d}}:\left(n_{\mathrm{SPP}}-n_{d}\right) / n_{d} \simeq \epsilon_{d}^{2} / \epsilon_{m}^{2} \ll 1$, strongly diminishing the perspectives of subdiffraction light confinement with an isolated SPP wave.

The light confinement with plasmonic layers can be significantly improved in multilayer structures. When two metal-dielectric interfaces are brought close to each other, the SPP waves propagating on individual interfaces couple 


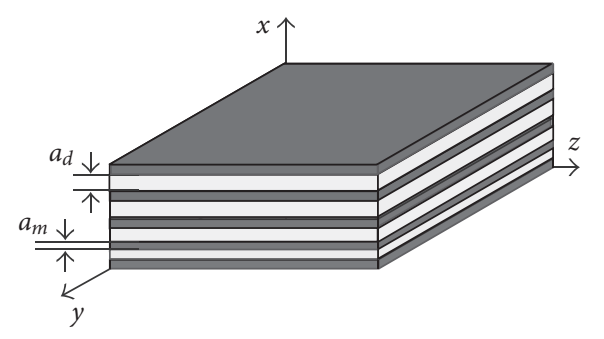

FIGURE 1: Schematic of a multilayered composite. $a_{m}$ and $a_{d}$ are the thicknesses of plasmonic (metallic) and dielectric composites, respectively.

together. Similar to quantum-mechanical coupling between modes of two quantum wells, which leads to the formation of symmetric and antisymmetric wavefunctions, the coupling of two SPPs yields the formation of symmetric and antisymmetric SPP combinations. This SPP coupling is accompanied by splitting between their propagation constants, increasing the effective index of one of the modes relative to that of an isolated SPP. In multilayered composites, the modes on each metal-dielectric interface couple to each other, leading to formation of free-space-like supermodes [25]. In the end, the multilayer metal-dielectric structure behaves like a strongly-anisotropic homogeneous metamaterial, with applications ranging from nonmagnetic negative index structures to optical links between nano- and micro-scaled systems $[9,12,26]$. The goals of this work are to provide a comprehensive study of the evolution of electromagnetic properties of plasmonic multilayer composites from an isolated SPP to effective-medium regime, to assess the perspectives of utilizing multilayer systems for nanoplasmonic applications and to present a detailed analysis of the validity of nonlocal effective-medium theory, originally proposed in [27].

\section{APPROACH}

To gain access into the physics behind the optical properties of nanoplasmonic structures and to verify the accuracy of our analytical results, we have employed the numerical solutions of Maxwell equations via transfer matrix method (TMM) $[28,29]$.

In this technique, the material is represented as a set of homogeneous layers. The solutions of Maxwell equations in each layer yield the set of modes; these are plane wavespropagating or evanescent-in the case of infinite layers, cylindrical waves in case of layers inside circular waveguides (optical fibers), and so forth. Boundary conditions are then used to relate the vector of amplitudes of modes in a given ( $j$ th) layer $b_{j}$ to the modes in the neighboring layer. These linear relationships are then expressed in terms of the transfer matrix

$$
b_{j+1}=\widehat{T}_{j+1, j} b_{j} .
$$

Thus, differential Maxwell equations are essentially reduced to linear equations for transfer matrices. Note that layerspecific transfer matrices can be multiplied together yielding the single matrix which describes the collective behavior of the multilayer composite.

The TMM technique is in principle exact and provides a complete solution of Maxwell equations. In particular, TMM solutions can be used to find the field propagation through an arbitrary layered material, to find the dispersion characteristics of supermodes of layered systems, and to find the "microscopic" field distribution in these supermodes. The solutions of latter problems typically involve finding eigenvalues and eigenvectors for collective (composite-level) transfer matrices. However, these exact numerical solutions are extremely computationally intensive and require an increase in precision past standard machine precision for even relatively small number of layers.

\section{RESULTS AND DISCUSSION}

In this main section of the manuscript, we first describe the evolution of the coupled SPP modes as we increase the number of layers in the multilayer structures. We show that depending on the relationship between $\epsilon_{m}$ and $\epsilon_{d}$, the supermodes may have positive or negative refractive indices. As the number of layers in the composite is increased, the structure of the supermodes resembles that of plane waves propagating through the homogeneous strongly anisotropic composite. We derive the analytical relationship describing the effective permittivity tensor of such a media and provide several applications of multilayer structures for nm-scale light management.

\subsection{Evolution of SPP modes in multilayer structures}

We start from the analysis of mode evolution as the function of the number of layers in the system. For illustrative purposes, in the remaining of the paper, we focus on the optical response of metal-dielectric composites; we note, however, that our arguments are directly applicable to the IR (or THz) response of doped semiconductors exhibiting plasma behavior, as well as to other materials with negative permittivities, including $\mathrm{SiC}$ and other polar dielectrics. The potential applications at $\mathrm{THz}$ frequencies include biology and medical sciences, homeland security, global environmental monitoring, and ultrafast computing [30].

A single metal-dielectric interface supports an SPP. When two metal layers are brought together, the SPP modes propagating on their interfaces interact with each other, leading to the formation of symmetric and antisymmetric SPP combinations as shown in Figure 2. The interaction of isolated SPP modes also leads to the splitting between effective indices of their symmetric and antisymmetric combinations. As the separation between metal films is reduced, the splitting between modal indices grows. Eventually, the antisymmetric mode experiences a cutoff, and only symmetric mode (known as gap plasmon [31]) survives.

The four-interfaced "sandwich" system formed by a thin metal film inserted into dielectric space between two metal cladding regions in principle supports four SPP combinations. However, if such a film is brought close enough to one 

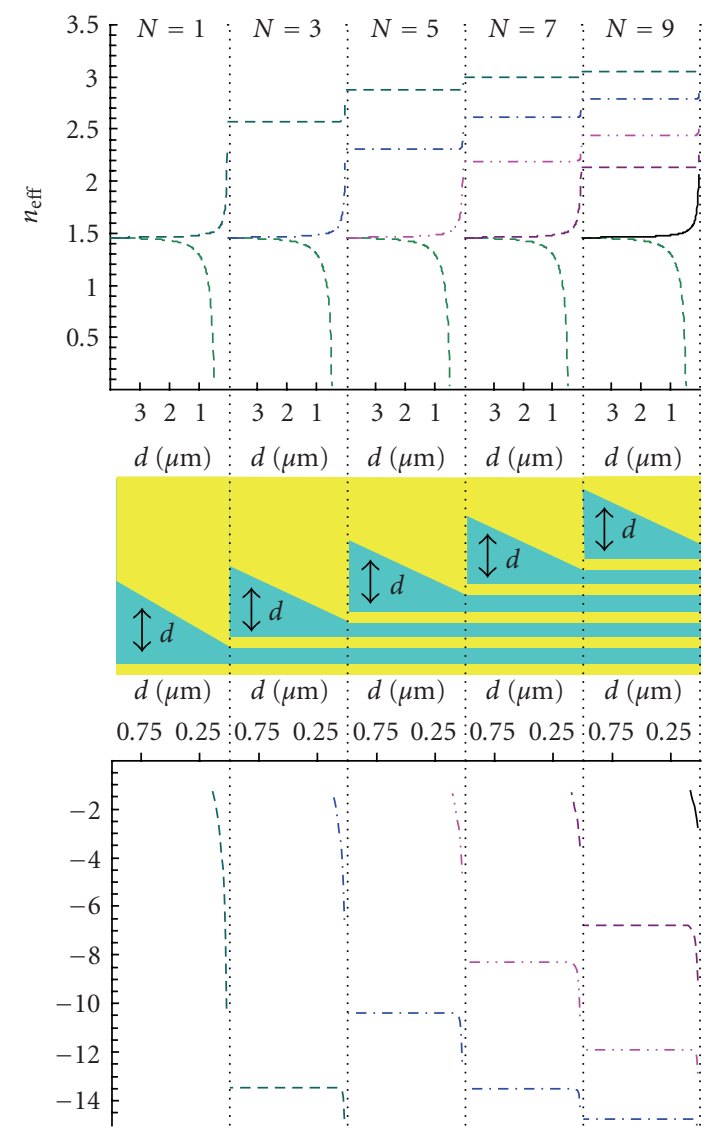

FIgURE 2: The evolution and splittings of coupled SPP modes propagating along $z$-direction (see Figure 1 ); $n=k_{z} c / \omega$ at $\lambda_{0}=$ $\omega / c=1.55 \mu \mathrm{m}$ is shown. The top part of the figure corresponds to "positive-index" structure: $\epsilon_{d}=1.444^{2} ; \epsilon_{m}=-114.5+11.01 i$; the bottom part describes a "negative-index" one: $\epsilon_{d}=1.444^{2}$; $\epsilon_{m}=-1+0.1 i$; the inset schematically shows the $x z$-crossection of the system; final layer thickness is $25 \mathrm{~nm}$.

of the cladding regions, the total number of modes is reduced to three; further confinement of the structure reduces the total number of modes to two-the symmetric and antisymmetric combinations of gap plasmons. Note that the indices of these modes continue to repulse from each other, yielding a formation of the modes with effective indices exceeding the one of the isolated gap plasmon. Each additional metal strip inserted into this sandwich system will effectively lead to the formation of an additional supermode. Thus, a total number of modes hosted by the metal-dielectric multilayer system surrounded by two metallic cladding regions will be equal to the number of dielectric layers in the system (Figure 2).

A similar process of mode formation takes place in the layered metamaterial when the excitation frequency is close to the plasma frequency of its plasmonic component $\left(\left|\epsilon_{m}\right|<\right.$ $\left.\epsilon_{d}\right)$. While an isolated metal interface does not support an SPP in this case, two-interface system supports a single antisymmetric mode with negative refraction index $[12,32]$. In a sense, the behavior of the metal-dielectric-metal system for $\left|\epsilon_{m}\right|<\epsilon_{d}$ is exactly opposite to that of $\left|\epsilon_{m}\right|>\epsilon_{d}$. The signs

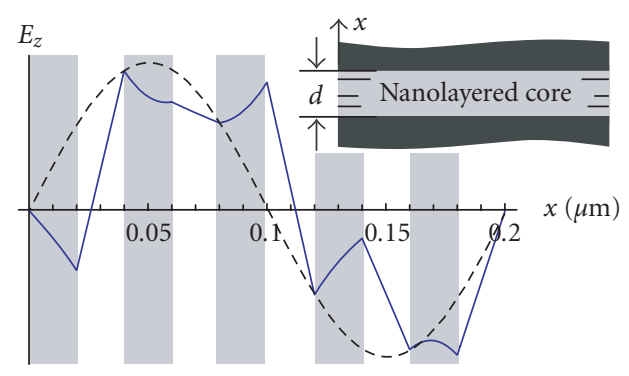

(a)

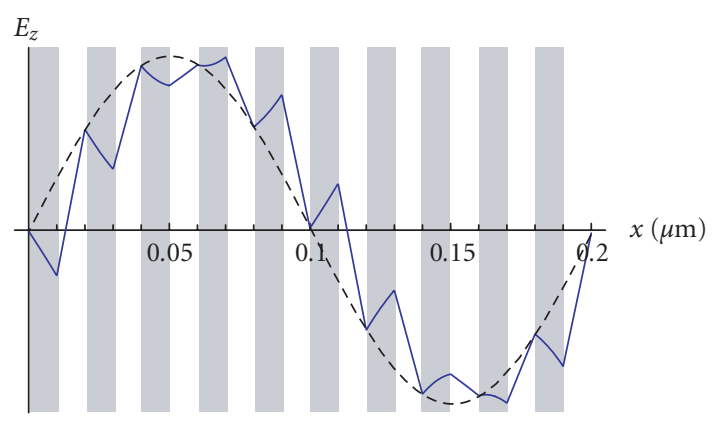

(b)

FIGURE 3: Field structure of a $\mathrm{TM}_{1}$ mode of planar waveguide formed by metal-dielectric stack between two perfectly conducting metal claddings for a number of layers $N=10$ (a) and $N=20$ (b); $\epsilon_{m}=-100 ; \epsilon_{d}=2 ; \lambda_{0}=1.55$ [27].

of their refractive indices are opposite to each other; furthermore, the cutoff of the mode in one of the systems can be related to the appearance of the mode in the other one. The multilayered composite with $\left|\epsilon_{m}\right|<\epsilon_{d}$ with $N$ dielectric layers supports a total of $N$ negative-index supermodes. The evolution of these supermodes is illustrated in Figure 2.

As the number of layers increases, so does the number of supermodes. At any given frequency, the composite with a very large number of layers essentially supports a continuum of modes with different modal structure. Some of these modes in the composites with 10 and 20 layers between two perfectly metallic claddings are shown in Figure 3. Note that the structure of these modes strongly resembles that of standing waves. This is obviously not surprising: the properties of nanostructured composites are expected to follow effectivemedium theory (EMT) predictions.

The exact process of convergence to the effective-medium regime is of a great interest. An analytical description of this process could be used to explain the experiments with a few metal layers $[25,32]$ or more importantly to predict the behavior of larger multilayer composites based on experiments with few layers. Figure 4 shows the dependence of the effective modal index in the nanolayered system (Figure 1) as the function of the number of metal layers. One can see that even when the number of layers is relatively large $(\gtrsim 10)$ and even when the individual layer thickness is much smaller than the wavelength, the properties of multilayer stack still significantly deviate from the EMT. As explained in [27], the origin 


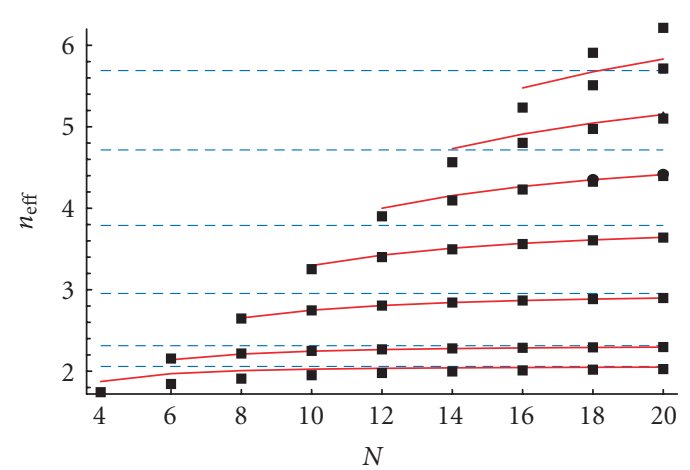

(a)

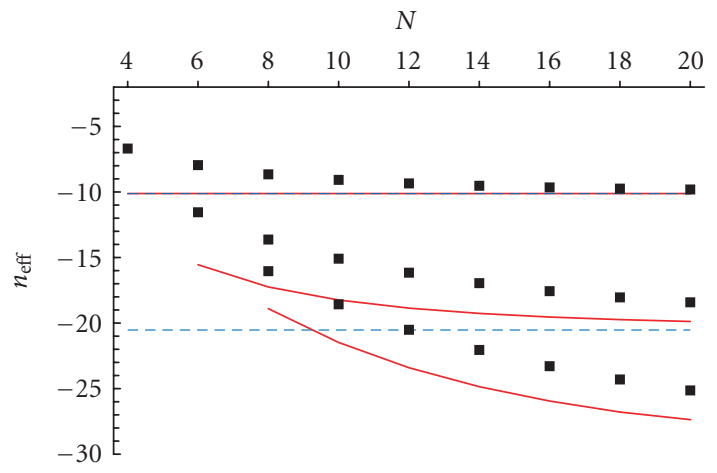

(c)

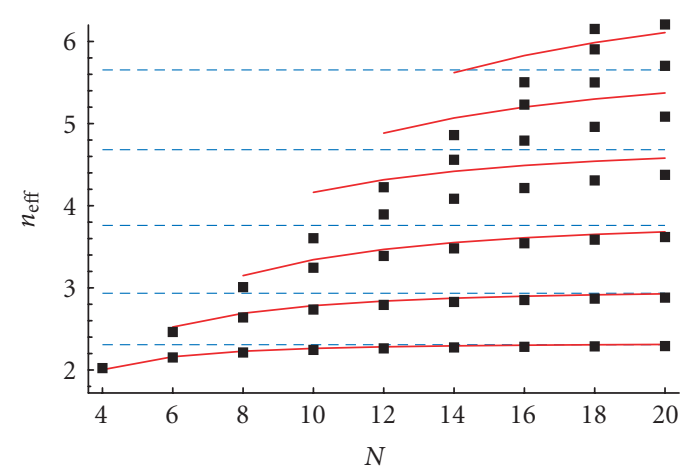

(b)

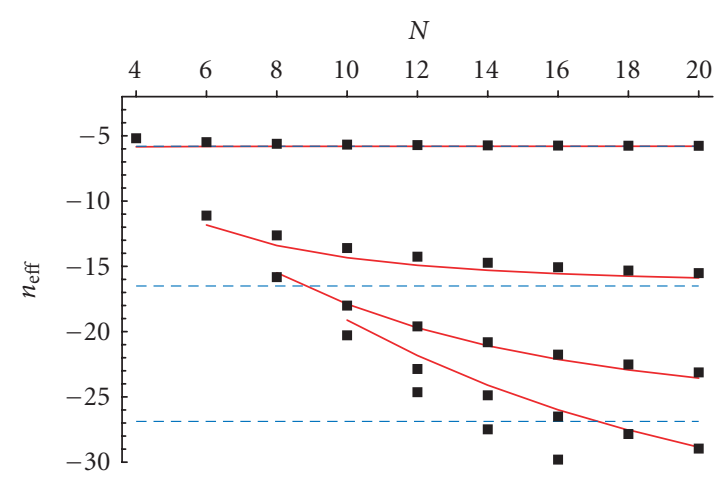

(d)

FIGURE 4: The comparison between effective modal indices of TM modes of 200-nm-thick planar waveguides with perfectly conducting (a), (c) and vacuum (b), (d) claddings plotted as a function of a number of layers in the metamaterial waveguide core $N$; material parameters are $\epsilon_{m}=-114.5+11.01 i ; \epsilon_{d}=1.444^{2}\left(\mathrm{Au}-\mathrm{SiO}_{2}\right.$ composite) (a), (b) and $\epsilon_{m}=-1+0.1 i ; \epsilon_{d}=1.444^{2}$ (c), (d); $\lambda_{0}=1.55 \mu \mathrm{m}$; solid lines, dashed lines, and symbols correspond to local EMT, nonlocal EMT, and TMM results, respectively; due to extremely large effective indices, $|n|$ nonlocal EMT is not applicable for higher-order modes in (c), (d).

of this relatively slow convergence lies in the strong field variation across individual layers of the system. Such a field variation leads to the strong dependence of the effective permittivity on the modal structure across the layers or-since the waveguide modes are typically "standing" equivalents of "propagating" bulk waves-on the direction of field propagation through the nanolayered metamaterials. The dependence of permittivity on components of wavevector, known as spatial dispersion, often appears in homogeneous media when the scale of field variation becomes comparable to interatomic distances. The onset of spatial dispersion in metamaterials can be typically related to substantial field variation on the interparticle ("meta-atom") scale. The spatial dispersion has been recently found in $\mathrm{GHz}$ nanowire composites $[20,33,34]$; it was later shown that response of optical nanowire structures is substantially different from their lowfrequency counterparts [11]. Using a straightforward fieldmatching technique [27], we arrive at the following expression for effective permittivity of nanolayered composites:

$$
\begin{aligned}
\epsilon_{x}^{\mathrm{eff}} & =\frac{\epsilon_{x}^{(0)}}{1-\delta_{x}(k, \omega)}, \\
\epsilon_{y z}^{\mathrm{eff}} & =\frac{\epsilon_{y z}^{(0)}}{1-\delta_{y z}(k, \omega)},
\end{aligned}
$$

where

$$
\begin{aligned}
& \epsilon_{x}^{(0)}=\frac{\left(a_{m}+a_{d}\right) \epsilon_{m} \epsilon_{d}}{a_{d} \epsilon_{m}+a_{m} \epsilon_{d}}, \\
& \epsilon_{y z}^{(0)}=\frac{a_{m} \epsilon_{m}+a_{d} \epsilon_{d}}{a_{m}+a_{d}},
\end{aligned}
$$

and the nonlocal corrections are given by

$$
\begin{aligned}
\delta_{x} & =\frac{a_{m}^{2} a_{d}^{2}\left(\epsilon_{d}-\epsilon_{m}\right)^{2} \epsilon_{x}^{(0)^{2}}}{12\left(a_{m}+a_{d}\right)^{2} \epsilon_{m}^{2} \epsilon_{d}^{2}}\left(\epsilon_{y z}^{(0)} \frac{\omega^{2}}{c^{2}}-\frac{k_{x}^{2}\left(\epsilon_{m}+\epsilon_{d}\right)^{2}}{\epsilon_{y z}^{(0)}}\right), \\
\delta_{y z} & =\frac{a_{m}^{2} a_{d}^{2}\left(\epsilon_{d}-\epsilon_{m}\right)^{2}}{12\left(a_{m}+a_{d}\right)^{2} \epsilon_{y z}^{(0)}} \frac{\omega^{2}}{c^{2}} .
\end{aligned}
$$

Note that the choice of $\omega / c$ and $k_{x}$ to describe the nonlocality in (5) as opposed to $k_{y}$ or $k_{z}$ is somewhat arbitrary since in the EMT regime, the components of the wavevector are related to each other via

$$
\begin{aligned}
& \frac{\omega^{2}}{c^{2}}=\frac{k_{x}^{2}}{\epsilon_{y z}^{\mathrm{eff}}}+\frac{k_{y}^{2}+k_{z}^{2}}{\epsilon_{x}^{\mathrm{eff}}} \\
& \frac{\omega^{2}}{c^{2}}=\frac{k_{x}^{2}+k_{y}^{2}+k_{z}^{2}}{\epsilon_{y z}^{\mathrm{eff}}}
\end{aligned}
$$




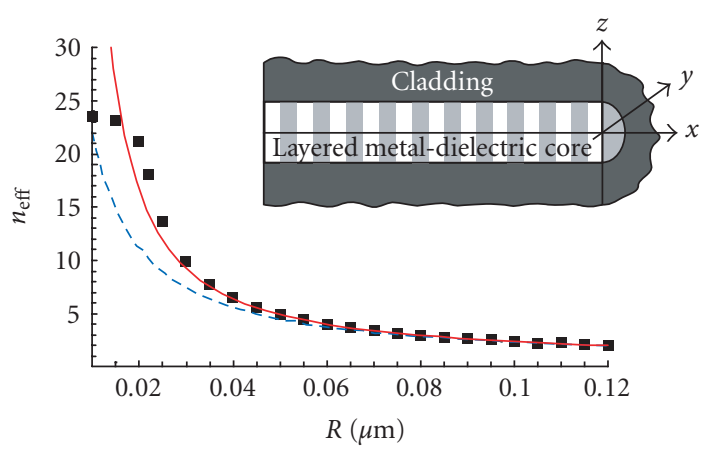

(a)

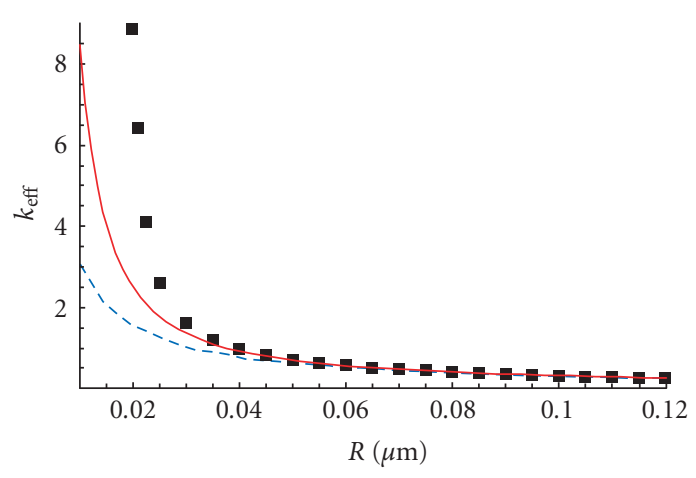

(c)

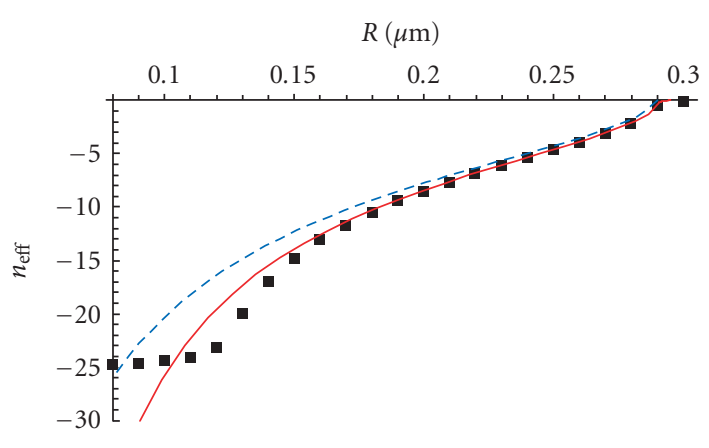

(b)

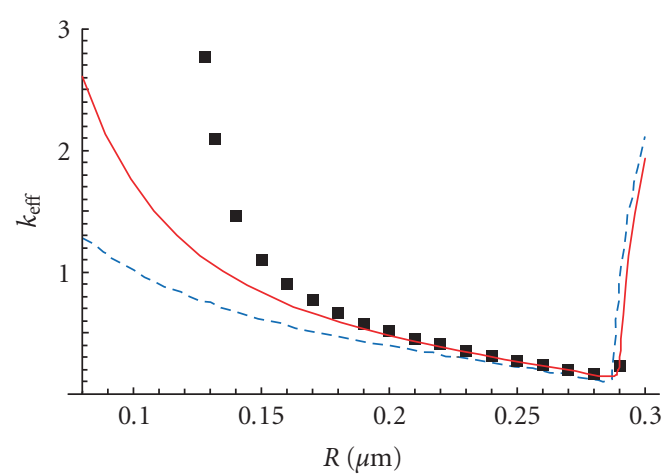

(d)

FIGURE 5: The comparison between effective refractive indices $n_{\text {eff }}=\operatorname{Re}\left(k_{x} c / \omega\right)$ (a), (b) and extinction coefficients $k_{\text {eff }}=\operatorname{Im}\left(k_{x} c / \omega\right)(\mathrm{c}),(\mathrm{d})$ of $\mathrm{TM}_{01}$ modes in cylindrical waveguides with multilayer cores and perfectly conducting claddings (inset); dashed lines, solid lines and dots correspond to results of local EMT, nonlocal EMT, and exact solution of the dispersion equation [28] respectively. The multilayered core is composed of 15-nm layers with (a), (c) $\epsilon_{m}=-1+0.1 i ; \epsilon_{d}=1.444^{2}$, and (b), (d) $\epsilon_{m}=-114.5+11.01 i ; \epsilon_{d}=1.444^{2}\left(\mathrm{Au}-\mathrm{SiO} \mathrm{O}_{2}\right.$ structure); $\lambda_{0}=1.55 \mu \mathrm{m}$; similar agreement between nonlocal EMT and exact dispersion equation is achieved for cylindrical systems with air claddings; note that the signs of refractive indices are opposite to those for planar mode propagation (see Figure 4).

In this section, we assume the propagation along the $z$-axis $\left(k_{y}=0\right)$ with waveguide modes in $x$-direction; for perfectly conducting waveguide walls, $k_{x}=\pi \mathrm{m} / d$ with $d$ being waveguide thickness and $m$ being integer mode number. For waveguide with realistic walls, $k_{x}$ could be found using "local" EMT approximation (4) or in self-consistent manner using $(4), \ldots,(6)$.

We stress that the validity of EMT response requires the typical scale of field variation to be much larger than any structural size of the system. For nanolayered composite, such a requirement results in an additional condition $\left|k_{x}\left(a_{m}+a_{d}\right)\right| \ll 1$, which in some cases is much stricter than a commonly used criterion $\left(a_{m}+a_{d}\right) \omega / c \ll 1$. Note that the nonlocal effects mostly (see discussion below (5)) affect the $x$-component of material permittivity. Since our metamaterial is completely homogeneous in $y z$-plane, such a result is a quite expected one.

The agreement between the developed nonlocal EMT and exact solutions of Maxwell equations using TMM are shown in Figure 4. For illustrative purposes, we concentrate on TM modes; TE waves are not affected by the anisotropy, and are typically not affected by nonlocal effects. It is clearly seen that nonlocal EMT adequately describes the behavior of the system. As expected, the agreement between EMT and exact (TMM) solutions of Maxwell equations worsens for higher-order modes where $\left|k_{x}(a+b)\right| \gtrsim 1$. Since the components of wavevector are related through (6), this validity condition is typically similar to $n_{\text {eff }} \gg 1$. Increasing the total thickness of the structure (decreasing $k_{x}$ ) or reducing the typical layer size or the number of layers restores the agreement.

The behavior of multilayers with $\left|\epsilon_{m}\right|<\epsilon_{d}$, but same sign of $\epsilon_{y z}^{(0)}$ and $\epsilon_{x}^{(0)}$, is an interesting one. The EMT regime of these systems does not originate from coupling of individual left-handed SPPs. As follows from our numerical solutions of Maxwell equations, reduction of layer thickness in these structures is accompanied by the increase of modal indices of SPP supermodes, so that the condition of EMT validity is never met. However, substantially thick multilayered structures with positive $\epsilon_{x}^{(0)}, \epsilon_{y z}^{(0)}$ support right-handed "volume" modes, described by the EMT.

Note that as explained above, multilayered system supports a number of modes with $n_{\text {eff }}=\operatorname{Re}\left(k_{z} c / \omega\right) \gg 1$, opening the perspectives for subwavelength light manipulation in 


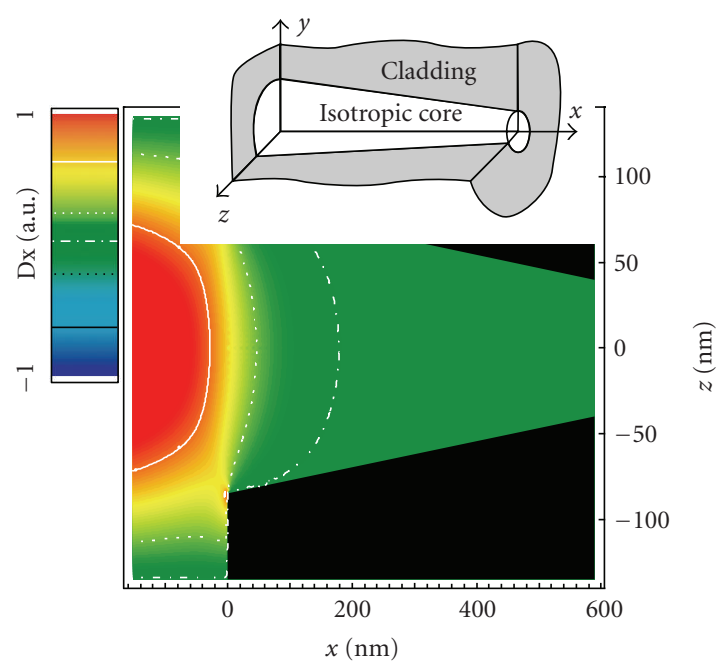

(a)

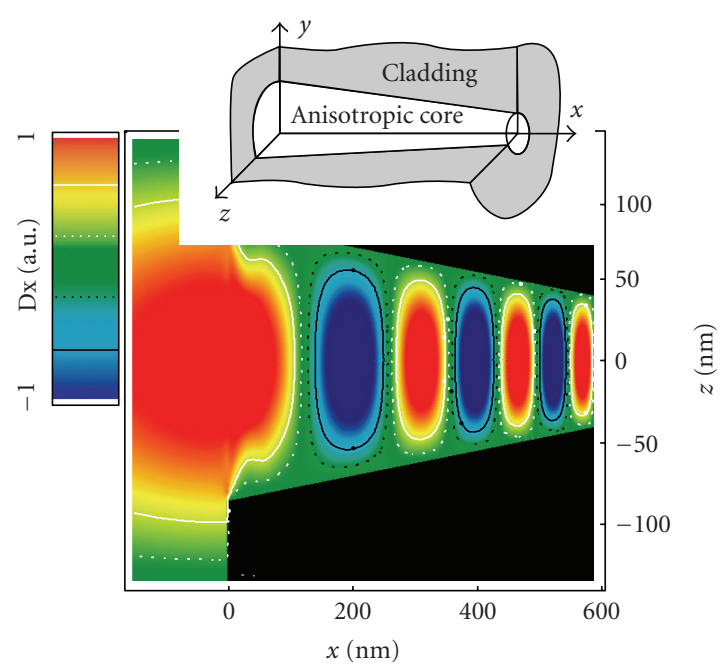

(b)

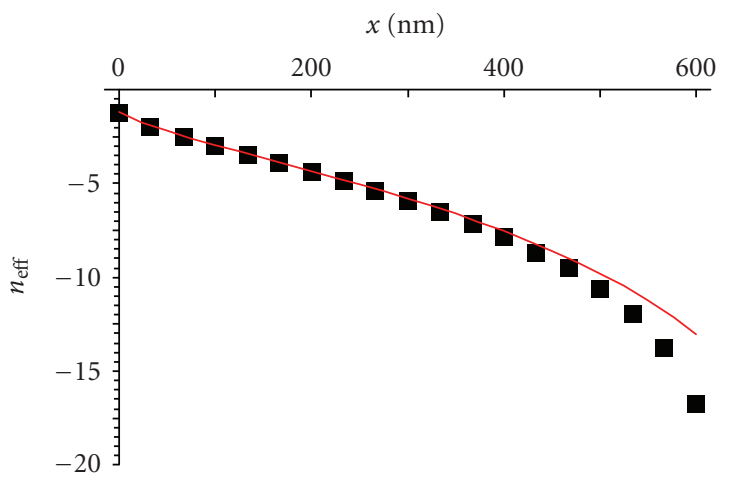

(c)

FIgURE 6: Mode propagation in conventional waveguide (a) and in anisotropy-based photonic funnel (b); material parameters $\epsilon_{m}=$ $-73.02+1.64 i(\mathrm{Ag}), \epsilon_{d}=12(\mathrm{Si}), a_{m}=a_{d}=15 \mathrm{~nm}$, and $\lambda_{0}=1.2 \mu \mathrm{m}$, and the funnel design resembles those in [9]; panel (c), which shows the effective modal index along the funnel in (b), demonstrates the physics behind nanoscale mode compression; symbols and solid lines represent TMM results and nonlocal EMT, respectively. planar structures with highly-confined optical modes with positive or negative refractive index [35]. The physics behind the existence of these highly confined modes can be illustrated using the following picture: the TM modes of a waveguide with strongly anisotropic dielectric core do not have a cutoff. As the waveguide size $d$ is reduced, the effective modal index grows inversely proportional to $d$ (see $[9-11,26]$ for details). Correspondingly, the internal wavelength is reduced $\left(\lambda_{\text {in }} \propto d\right)$, postponing the onset of diffraction limit. Spatial dispersion, appearing in metamaterial response changes the effective-medium parameters, reducing the effective anisotropy, and eventually leading to a cutoff of waveguide modes.

\subsection{Subdiffraction light propagation in nanolayer-filled waveguides}

Although planar nanolayer systems are capable of guiding the light in deep subwavelength areas and may be used to realize optical packet management in 2D geometries, these materials cannot be directly coupled to optical fibers.

Ideally, two conditions must be met to achieve such a coupling between microscale optical fiber and nanoscale systems (planar waveguides, molecules, quantum dots, etc.). First, the coupler waveguide (fiber) should be able to support confined modes as waveguide radius is reduced to the nanoscale. Second, the spatial profile of a mode in the coupler waveguide should be similar to the one of the mode in optical fiber. Both these conditions are fulfilled in waveguides with strongly anisotropic cores when the axis of optical anisotropy is coaligned with direction of mode propagation.

One of the ways to achieve the required strong anisotropy of dielectric permittivity relies on multilayered nanoplasmonic structures. To coalign the optical axis with direction of mode propagation, we propose to build a waveguide with a multilayer dielectric core as shown in Figure 5 [9]. Similar to the planar structure described above, such a circular waveguide essentially does not have a cutoff radius in the "local EMT" regime, where effective modal index $n_{\mathrm{eff}}=$ $k_{x} c / \omega \propto 1 / R$, postponing the appearance of diffraction limit (see [9] for details). The appearance of the cutoff can be once again related to the effective nonlocalities of metamaterial structures (Figures 5(c), 5(d)). Note however, that due to strong anisotropy of effective permittivity the positive-index modes propagating along $y z$-plane correspond to negativeindex modes propagating along $x$-direction and vice versa [36].

The comparison between the effective modal index of a circular waveguide with multilayer core, calculated using exact approach [28], local, and nonlocal EMTs, is shown in Figure 5. The performance of such a system is illustrated in Figure 6, where the mode propagation in waveguide with nanolayered core is compared to the mode propagation in waveguide with conventional isotropic core. The appearance of the cutoff in isotropic system and the absence of the cutoff in tapered anisotropic waveguide (photonic funnel [9]) are clearly seen. 


\section{CONCLUSIONS}

In conclusion, we analyzed the behavior of coupled SPP modes in multilayered metal-dielectric materials. Using exact numerical solutions of Maxwell equations, we have shown that as the number of layers increases, the optical properties of coupled SPP oscillations approach the behavior of strongly anisotropic homogeneous uniaxial media, and analyzed the validity of new analytical description of effective permittivity of such a material-nonlocal effective-medium theory. Finally, we illustrated the applications of multilayered structures for subdiffraction light propagation in planar and circular waveguides and for coupling between micro-scale and nanoscale systems.Our results, although presented here for optical response of metal-dielectric composites, are applicable to any layered structures composed from oppositepermittivity materials, including $\mathrm{UV}$ response of $\mathrm{Al}$ composites and IR and $\mathrm{THz}$ responses of doped semiconductors and polar dielectrics.

\section{ACKNOWLEDGMENTS}

This research was partially supported by PRF (ACS), ARO, and ONR.

\section{REFERENCES}

[1] J. G. Goodberlet, "Patterning $100 \mathrm{~nm}$ features using deepultraviolet contact photolithography," Applied Physics Letters, vol. 76, no. 6, pp. 667-669, 2000.

[2] H. Schmid, H. Biebuyck, B. Michel, and O. J. F. Martin, "Lightcoupling masks for lensless, sub-wavelength optical lithography," Applied Physics Letters, vol. 72, no. 19, pp. 2379-2381, 1998.

[3] A. Lewis, H. Taha, A. Strinkovski, et al., "Near-field optics: from subwavelength illumination to nanometric shadowing," Nature Biotechnology, vol. 21, no. 11, pp. 1378-1386, 2003.

[4] E. Betzig, J. K. Trautman, T. D. Harris, J. S. Weiner, and R. L. Kostelak, "Breaking the diffraction barrier: optical microscopy on a nanometric scale," Science, vol. 251, no. 5000, pp. 14681470, 1991.

[5] H. Rong, Y.-H. Kuo, S. Xu, et al., "Monolithic integrated Raman silicon laser," Optics Express, vol. 14, no. 15, pp. 67056712, 2006.

[6] A. W. Fang, H. Park, O. Cohen, R. Jones, M. J. Paniccia, and J. E. Bowers, "Electrically pumped hybrid AlGaInAs-silicon evanescent laser," Optics Express, vol. 14, no. 20, pp. 92039210, 2006.

[7] S. A. Maier, P. G. Kik, H. A. Atwater, et al., "Local detection of electromagnetic energy transport below the diffraction limit in metal nanoparticle plasmon waveguides," Nature Materials, vol. 2, no. 4, pp. 229-232, 2003.

[8] A. Karalis, E. Lidorikis, M. Ibanescu, J. D. Joannopoulos, and M. Soljačić, "Surface-plasmon-assisted guiding of broadband slow and subwavelength light in air," Physical Review Letters, vol. 95, no. 6, Article ID 063901, 4 pages, 2005.

[9] A. A. Govyadinov and V. A. Podolskiy, "Metamaterial photonic funnels for subdiffraction light compression and propagation," Physical Review B, vol. 73, no. 15, Article ID 155108, 5 pages, 2006.

[10] A. A. Govyadinov and V. A. Podolskiy, "Gain-assisted slow to superluminal group velocity manipulation in nanowaveg- uides," Physical Review Letters, vol. 97, no. 22, Article ID 223902, 2006.

[11] J. Elser, R. Wangberg, V. A. Podolskiy, and E. E. Narimanov, "Nanowire metamaterials with extreme optical anisotropy," Applied Physics Letters, vol. 89, no. 26, Article ID 261102, 3 pages, 2006.

[12] A. Alù and N. Engheta, "Optical nanotransmission lines: synthesis of planar left-handed metamaterials in the infrared and visible regimes," Journal of the Optical Society of America B, vol. 23, no. 3, pp. 571-583, 2006.

[13] J. B. Pendry, "Negative refraction makes a perfect lens," Physical Review Letters, vol. 85, no. 18, pp. 3966-3969, 2000.

[14] N. Fang, H. Lee, C. Sun, and X. Zhang, "Sub-diffractionlimited optical imaging with a silver superlens," Science, vol. 308, no. 5721, pp. 534-537, 2005.

[15] G. Shvets and Y. A. Urzhumov, "Engineering the electromagnetic properties of periodic nanostructures using electrostatic resonances," Physical Review Letters, vol. 93, no. 24, Article ID 243902, 4 pages, 2004.

[16] D. O. S. Melville and R. J. Blaikie, "Super-resolution imaging through a planar silver layer," Optics Express, vol. 13, no. 6, pp. 2127-2134, 2005.

[17] M. I. Stockman, "Nanofocusing of optical energy in tapered plasmonic waveguides," Physical Review Letters, vol. 93, no. 13, Article ID 137404, 4 pages, 2004.

[18] J. B. Pendry, A. J. Holden, W. J. Stewart, and I. Youngs, "Extremely low frequency plasmons in metallic mesostructures," Physical Review Letters, vol. 76, no. 25, pp. 4773-4776, 1996.

[19] A. K. Sarychev, R. C. McPhedran, and V. M. Shalev, "Electrodynamics of metal-dielectric composites and electromagnetic crystals," Physical Review B, vol. 62, no. 12, pp. 8531-8539, 2000.

[20] P. A. Belov and C. R. Simovski, "Homogenization of electromagnetic crystals formed by uniaxial resonant scatterers," Physical Review E, vol. 72, no. 2, Article ID 026615, 15 pages, 2005.

[21] M. A. Shapiro, G. Shvets, J. R. Sirigiri, and R. J. Temkin, "Spatial dispersion in metamaterials with negative dielectric permittivity and its effect on surface waves," Optics Letters, vol. 31, no. 13, pp. 2051-2053, 2006.

[22] E. M. Lifshitz and L. P. Pitaevskii, Physical Kinetics, vol. 10 of Course of Theoretical Physics, Reed Educational and Professional Publishing, Oxford, UK, 1984.

[23] With a few exceptions $(\mathrm{Ag}, \mathrm{Al})$, the response of metals in the $\mathrm{UV}$ and visible frequency ranges is also strongly affected by interband transitions.

[24] L. D. Landau, E. M. Lifshitz, and L. P. Pitaevskii, Electrodynamics of continuous media, vol. 8 of Course of Theoretical Physics, Reed Educational and Professional Publishing, Oxford, UK, 2nd edition, 1984.

[25] I. Avrutsky, I. Salakhutdinov, J. Elser, and V. A. Podolskiy, "Highly confined optical modes in nanoscale metaldielectric multilayers," Physical Review B, vol. 75, no. 24, Article ID 241402, 4 pages, 2007.

[26] V. A. Podolskiy and E. E. Narimanov, "Strongly anisotropic waveguide as a nonmagnetic left-handed system," Physical Review B, vol. 71, no. 20, Article ID 201101, 4 pages, 2005.

[27] J. Elser, V. A. Podolskiy, I. Salakhutdinov, and I. Avrutsky, "Nonlocal effects in effective-medium response of nanolayered metamaterials," Applied Physics Letters, vol. 90, no. 19, Article ID 191109, 3 pages, 2007.

[28] P. Yeh, A. Yariv, and C. Hong, "Electromagnetic propagation in periodic stratified media. I. General theory," Journal of the Optical Society of America, vol. 67, no. 4, pp. 423-438, 1977. 
[29] I. Avrutsky, "Guided modes in a uniaxial multilayer," Journal of the Optical Society of America A, vol. 20, no. 3, pp. 548-556, 2003.

[30] M. Tonouchi, "Cutting-edge terahertz technology," Nature Photonics, vol. 1, no. 2, pp. 97-105, 2007.

[31] D. F. P. Pile and D. K. Gramotnev, "Adiabatic and nonadiabatic nanofocusing of plasmons by tapered gap plasmon waveguides," Applied Physics Letters, vol. 89, no. 4, Article ID 041111, 1 page, 2006.

[32] H. Shin and S. Fan, "All-angle negative refraction for surface plasmon waves using a metal-dielectric-metal structure," Physical Review Letters, vol. 96, Article ID 073907, 2006.

[33] A. L. Pokrovsky and A. L. Efros, "Electrodynamics of metallic photonic crystals and the problem of left-handed materials," Physical Review Letters, vol. 89, no. 9, Article ID 93901, 4 pages, 2002.

[34] A. L. Pokrovsky and A. L. Efros, "Nonlocal electrodynamics of two-dimensional wire mesh photonic crystals," Physical Review B, vol. 65, no. 4, Article ID 45110, 8 pages, 2002.

[35] The strong overlap of nm-confined modes with metallic component of nanolayered structure leads to significant reduction of propagation length of these waves. The typical propagation length of the mode is of the order of $1 \ldots 10 \mu \mathrm{m}$. This length can be improved by using Ag instead of $\mathrm{Au}$, or by selection other low-loss media. These losses can be also compensated by incorporation of gain into metamaterial structure[29].

[36] V. A. Podolskiy and E. E. Narimanov, "Comment on: allangle broadband negative refraction of metal waveguide arrays in the visible range: theoretical analysis and numerical demonstration," Physical Review Letters, vol. 98, no. 17, Article ID 179401, 2007. 

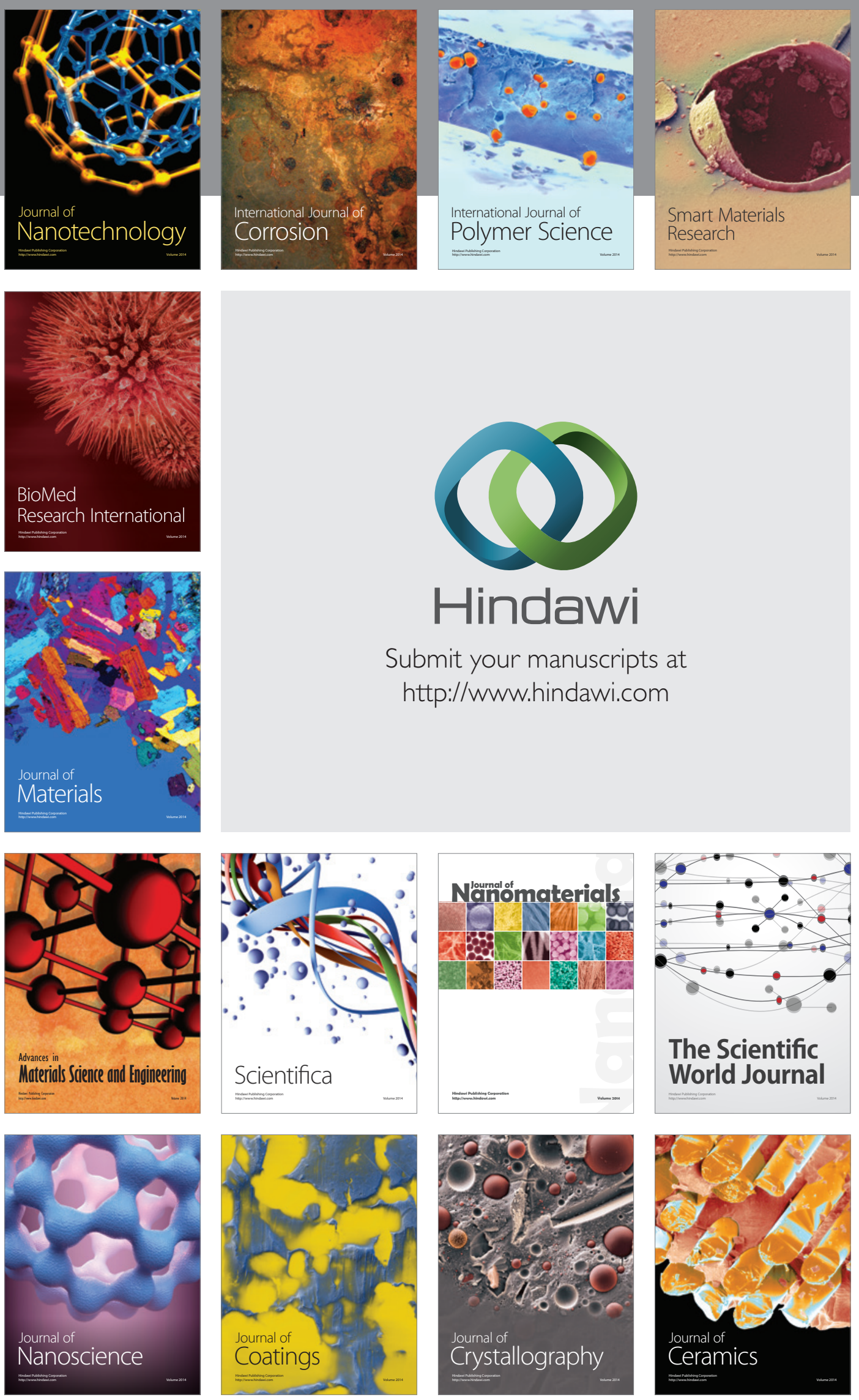

The Scientific World Journal

Submit your manuscripts at

http://www.hindawi.com

\section{World Journal}

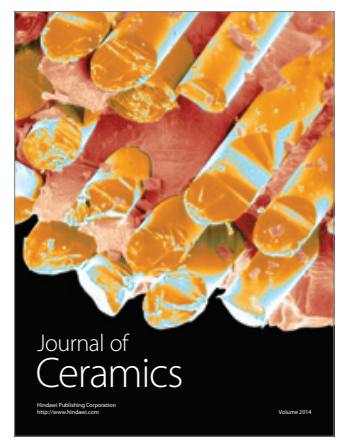

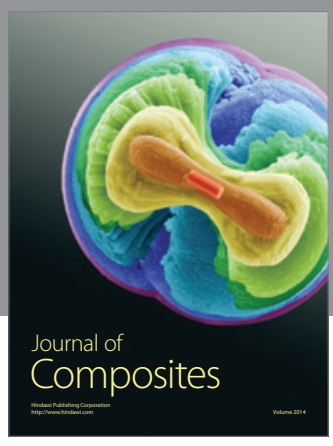
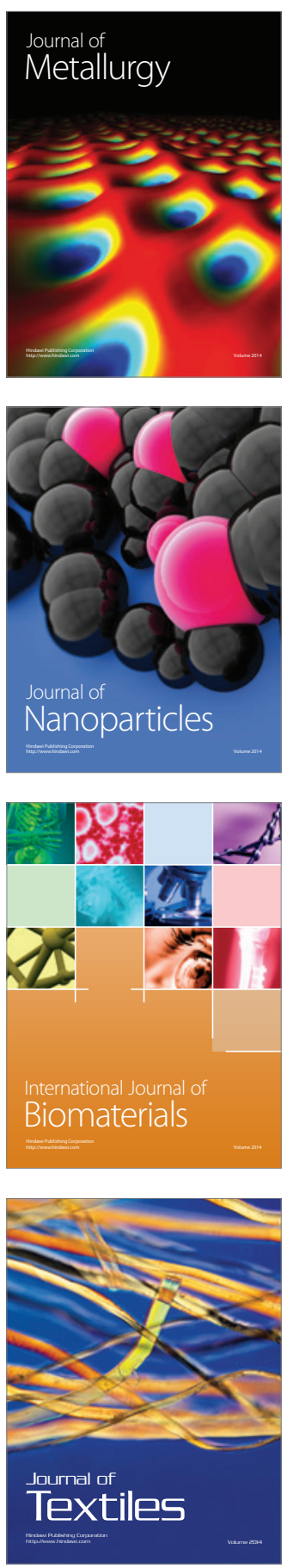